\title{
Permeability of multi-layer structures
}

\author{
Stanislav E. Solovyov, Anatoliy Ya. Goldman *
}

Department of Materials and Processing, Alcoa Closure Systems International, Inc. ATB, 1205 Elmore Street, Crawfordsville, IN 47933, USA; Fax 765-364-5678; Stanislav.Solovyov@alcoa.com, Anatoliy.Goldman@alcoa.com

(Received: April 14, 2004; published: April 27, 2004)

This work has been presented at the $11^{\text {th }}$ Annual POLYCHAR World Forum on Advanced Materials, January 7-10, 2003, in Denton, Texas, USA

\begin{abstract}
In this paper we show that 'permeability' of a heterogeneous structure with mass transport and thermodynamic properties varying across its thickness is a misleading concept leading to incorrect results and design decisions while two structural transmission rate equations are recommended for practical applications. The notion of structural identity of multi-layer films is introduced to explain the apparent failure of the 'permeability' concept. Structural identity of two or more films means the same material sequence in the structure relative to separated environments with constant relative thickness of each corresponding layer. Structurally identical films indeed have the same 'permeability', however the notion of identity is shown to contradict the practical goals of multi-layer film design. Engineering examples are provided to demonstrate potential misuses of the 'permeability' concept in practical multi-layer design decisions. Correct problem statements and calculation procedures are included. Some general limitations of transmission rate equations are also discussed. These include the role of boundary conditions, temperature and concentration dependence of permeant diffusivity and solubility in a polymer matrix, the presence of co-permeants, surface sorption effects, film thickness, homogeneity of polymer matrix for permeation purposes and correct utilization of available data for predicting gas transport properties of multilayer films.
\end{abstract}

\section{Introduction}

Prediction of water vapour and gas transmission rates through layered plastic structures is critical in design and manufacturing of barrier packaging. Unlike gas separation membranes used to efficiently separate gas mixture components, barrier packaging designs are intended to reduce mass transport rates of undesirable gases and vapours across the barrier and ultimately to extend the packaged product shelf life. Multi-layer packaging materials are predominantly produced as films and sheets via processes like co-extrusion and lamination to utilize unique performance properties of several materials in a single product. A recent paper by Cooksey et al. [1] summarized equations suggested in various package-engineering references for calculating permeability and transmission rates of atmospheric gases through multilayer films in steady state. It also recommended specific equations depending on 
desired applications and available experimental data. These equations formalized model calculations of transmission rate and 'permeability' of heterogeneous structures comprised of sequential homogeneous layers with uniform thicknesses and applied to specific permeating species.

Four types of equations were introduced: calculation of transmission rate of the heterogeneous structure from either transmission rates or permeabilities of individual layers, and calculation of 'permeability' of the structure from the same two data subsets. While the steady state transmission rate of a permeant across a heterogeneous structure when a fixed permeant pressure difference is imposed on opposite sides of the barrier is a valuable and universally accepted concept, the concept of 'permeability' of a heterogeneous structure is shown to not withstand close scrutiny. Also, when trying to apply the structural permeability equations to particular package design problems, the concept of 'permeability' of multi-layer structures causes confusion in interpretation of results within the packaging community. Clearly several clarifications are in order.

\section{Definitions}

Transmission rate (TR) also called permeance refers to the amount of permeant $q$ passed through the unit area $A$ of tested film sample in unit time $t$ under unit pressure difference $\Delta p$ of permeating species across the film thickness and specified conditions of temperature $T$ and relative humidity $\mathrm{RH}$ at the film surfaces (according to ASTM procedures D1434-82 for gases, D3985-02 for oxygen, E96-00e1 and F372-99 for water vapour in ref. [2]). Film thickness $L$ is not a part of $T R$ definition but is implied to be the film sample thickness. Permeability $P$ of material to specific permeant is its transmission rate normalized to a unit film thickness, usually 1 mil (= 0.001 inch $=25.4 \mu \mathrm{m}$ ) in the US. Thus, $T R$ and $P$ are formally defined as

$$
\begin{aligned}
& T R=\frac{q}{A t \Delta p} \\
& P=\frac{q L}{A t \Delta p}=T R \cdot L
\end{aligned}
$$

Units commonly used in package engineering in the United States for $P$ and $T R$ of gases are

Permeability $P: \quad \quad \quad \quad c(S T P) \cdot m i l /\left(100 \mathrm{in}^{2} \cdot 24 \mathrm{~h} \cdot \mathrm{atm}\right)$ at temperature $T$ and $\mathrm{RH}$

Transmission rate $T R: \quad \mathrm{cc}(\mathrm{STP}) /\left(100 \mathrm{in}^{2} \cdot 24 \mathrm{~h} \cdot \mathrm{atm}\right)$ at temperature $T$ and $\mathrm{RH}$

while corresponding SI units for gases are

Permeability $P$ (common): $\quad \mathrm{cm}^{3}(\mathrm{STP}) \cdot \mathrm{mm} \cdot \mathrm{m}^{-2} \cdot \mathrm{day}^{-1} \cdot \mathrm{bar}^{-1}$ at $T$ and $\mathrm{RH}$

(reference): $\quad \mathrm{m}^{3}(\mathrm{STP}) \cdot \mathrm{m} \cdot \mathrm{m}^{-2} \cdot \mathrm{s}^{-1} \cdot \mathrm{Pa}^{-1}$

Transmission rate TR (common): $\mathrm{cm}^{3}(\mathrm{STP}) \cdot \mathrm{m}^{-2} \cdot \mathrm{day}^{-1} \cdot \mathrm{bar}^{-1}$ at $T$ and $\mathrm{R}$

$$
\text { (reference): } \mathrm{m}^{3}(\mathrm{STP}) \cdot \mathrm{m}^{-2} \cdot \mathrm{s}^{-1} \cdot \mathrm{Pa}^{-1}
$$

Here the pressure difference in atm ( $1 \mathrm{~atm} \equiv 101.325 \mathrm{kPa})$, bar $(1 \mathrm{bar} \equiv 100 \mathrm{kPa})$, or $\mathrm{Pa}$ refers to the external pressures of the gaseous permeating species on the opposite sides of the membrane.

Permeability $P$ and transmission rate WVTR of water vapour are usually measured in grams of permeated water [3]: 
Permeability $P: \mathrm{g} \cdot \mathrm{mil} /\left(100 \mathrm{in}^{2} \cdot 24 \mathrm{~h}\right)$ at temperature $T$ and $\mathrm{RH}$ difference in air (or other carrier medium) at $1 \mathrm{~atm}$ of air pressure on both sides of the membrane

Transmission Rate WVTR: $\mathrm{g} /\left(100 \mathrm{in}^{2} \cdot 24 \mathrm{~h}\right)$ at temperature $T$ and $\mathrm{RH}$ difference in air at $1 \mathrm{~atm}$

The relative humidity difference across the membrane thickness is usually specified between some relative humidity value of atmospheric air (e.g., 60, 65, 75, 80, 85, 90, $100 \% \mathrm{RH}$ were reported in ref. [3]) and $0 \% \mathrm{RH}$ on the opposite side of the membrane. For concentration dependent diffusion when the diffusivity and TR of the permeant across a membrane depend on the local concentrations of the permeant dissolved in the membrane, both boundary conditions (absolute gas pressures or $\mathrm{RH}$ outside the membrane) must be specified. The same should be done in case of multicomponent diffusion when the presence of co-permeants affects the diffusion and TR of the permeant in question. The $\mathrm{RH}$ of permeating gas is included in the standardized TR measurement conditions for this very reason since many polar polymers are plasticized by water resulting in significant structural and corresponding permeant $T R$ changes. The most common example is ethylene-vinyl alcohol copolymer (EVOH) that exhibits very strong dependence of oxygen transmission rate (OTR) across it on $\mathrm{RH}$ of oxygen gas. To report OTR of EVOH when $\mathrm{RH}$ gradient is present across the membrane, $\mathrm{RH}$ at both surfaces of the membrane must be specified rather than just the $\mathrm{RH}$ difference since the data will differ dramatically for, say, $\mathrm{RH}$ pairs $0-40 \%$ and $60-100 \%$ corresponding to the same $\mathrm{RH}$ difference of $40 \%$ across the membrane.

We note that the amount of permeant $q$ is expressed in moles (mol) or as its mass in grams (g) used mostly for water vapour permeation, but for permanent gases in package engineering it is commonly referred to in $\mathrm{cc}\left(\mathrm{cm}^{3}\right)$ at STP, i.e., at $0^{\circ} \mathrm{C}$ and 1 atm of pressure.

\section{Equations}

Equations suggested for total transmission rate $T R_{T}$ and total permeability $P_{T}$ of a multi-layer structure with $N$ layers denoted $A, B, \ldots, N$ assuming homogeneity and uniform thickness of each layer are categorized in ref. [1] from package engineering refs. [4-7] and reproduced below. All these equations are derived from Fick's laws of diffusion assuming constant and equal permeant fluxes through all layers at steady state conditions and low concentrations of permeating species to invoke Henry's law on linear dependence of permeant partial pressure on its concentration. When concentration dependence of diffusion, nonlinearity of sorption isotherms and the effects of co-permeants on transport properties of materials can be neglected, the details of the derivation are trivial and can be found in most package engineering textbooks. We will only discuss the final equations.

\subsection{Calculating transmission rate from transmission rate data}

$$
T R_{T}=\frac{1}{\frac{1}{T R_{A}}+\frac{1}{T R_{B}}+\ldots+\frac{1}{T R_{N}}}
$$

This equation does not involve material permeabilities and layer thicknesses and does not lead to confusion in practice. Transmission rate of any particular layer is used regardless of uniformity of layer chemical composition and molecular structure. 
For example, layers $A$ and $B$ can be considered a single layer identified as $A B$ and $T R_{A B}$ is measured. Then we can write

$$
T R_{T}=\frac{1}{\frac{1}{T R_{A B}}+\frac{1}{T R_{C}}+\ldots+\frac{1}{T R_{N}}}
$$

If layers $A$ and $B$ are physically separate and uniform in their respective thickness and molecular structure, it is also true that

$$
T R_{A B}=\frac{1}{\frac{1}{T R_{A}}+\frac{1}{T R_{B}}}
$$

If materials $A$ and $B$ are blended however, then their molecular structure changes and Eq. (5) does not apply, but Eq. (4) is still valid, provided $T R_{A B}$ of the $A B$ blend is measured.

3.2 Calculating transmission rate from permeability data

$$
T R_{T}=\frac{1}{\frac{L_{A}}{P_{A}}+\frac{L_{B}}{P_{B}}+\ldots+\frac{L_{N}}{P_{N}}}
$$

Eq. (6) is essentially equivalent to Eq. (3) when each layer is made from a material of uniform chemical composition and molecular structure with known $P$.

\subsection{Calculating permeability from permeability data}

$$
P_{T}=\frac{L_{T}}{\frac{L_{A}}{P_{A}}+\frac{L_{B}}{P_{B}}+\ldots+\frac{L_{N}}{P_{N}}}
$$

Here the total structure thickness: $L_{T}=L_{A}+L_{B}+\ldots+L_{N}$.

\subsection{Calculating permeability from transmission rate data}

$$
P_{T}=\frac{L_{T}}{\frac{1}{T R_{A}}+\frac{1}{T R_{B}}+\ldots+\frac{1}{T R_{N}}}
$$

Eqs. (7) and (8) relating individual material $(P)$ and layer $(T R)$ data to permeability $P_{T}$ of multi-layer structure are the ones causing confusion in interpretation of results. The reason for that is an underlying assumption that it is possible to normalize the transmission rate of the whole structure to a unit thickness and thus obtain the structure 'permeability' according to permeability definition. Let us discuss the validity of this $T R$ normalization assumption.

\section{Structural identity}

In an ideal world, where we are able to vary any layer thickness within a structure at will and maintain the relative thickness of all layers constant, the TR normalization 
assumption is undoubtedly true. Constant relative thickness of layer $Z$ meaning $L_{Z} / L_{i}$ $=$ const for any $L_{i}$ as we vary $L_{z}$, i.e., thickness of all other layers $L_{i}$ along with the total thickness $L_{T}$ must change in proportion to $L_{z}$ change. We call the constant relative layer thickness property of multi-layer films a structural identity. Two or more films are structurally identical (broad definition) when exposed to identical pairs of distinct environments they separate, if they possess the same number and material sequence of layers relative to the separated environments as well as equal relative thickness of each corresponding layer. Strictly, for any layer number $Z$ in both films 1 and 2 numbered from the same external environment both films exposed to, it must be made from the same material $X$ having the same morphological structure and the relationship $L_{Z 1} / L_{T 1}=L_{Z 2} / L_{T 2}$ must be maintained for these films to be considered structurally identical in the given broad sense. Consider the following example of four multi-layer structures with corresponding layer thicknesses (arbitrary units) exposed to the identical pairs of distinct environments (say, fixed 'left' and 'right' environments):

Structure 1. Material sequence: A-B-C-A

Layer thickness: $\quad 4123 \quad$ Total: 10

Structure 2. Material sequence: A-B-C-A Layer thickness: $8246 \quad$ Total: 20

Structure 3. Material sequence: A-B-C-A

$$
\text { Layer thickness: } \quad 4223 \quad \text { Total: } 11
$$

Structure 4. Material sequence: A-B-A-C

$$
\text { Layer thickness: } \quad 4132 \quad \text { Total: } 10
$$

According to the given definition, films 1 and 2 are structurally identical even though their total thicknesses differ. On the other hand, pairs 1-3 and 2-3 are structurally different since the relative thickness of layer B in film 3 (and all other layers as well) is not the same as in film 1 or 2 , namely $L_{B} / L_{T}=1 / 10$ for films 1 and 2 while $L_{B} / L_{T}=2 / 11$ for film 3 . Film 4 is different from all others because it has a different material sequence. In fact, even mirror symmetrical films like

Structure 5. Material sequence: (Env. 1) A-B-C-D (Env. 2)

$$
\text { Layer thickness: } \quad 1234 \text { Total: } 10
$$

Structure 6. Material sequence: (Env. 1) D-C-B-A (Env. 2)

$$
\text { Layer thickness: } \quad 4321 \text { Total: } 10
$$

i.e., the same film with asymmetric layer structure exposed to the same distinct environments 1 and 2 will not in general exhibit the same mass transport behaviour when the material sequence of layers is reversed. The already mentioned effects including concentration dependent diffusion, multi-component diffusion with coupled co-permeant effects, and nonlinear sorption isotherms discussed later are responsible for this asymmetry. Hence, in general the structures 5 and 6 are not structurally identical in the broad sense when the environments they separate are taken into account, although in a narrow sense one can call them structurally identical from, say, manufacturing viewpoint when the surrounding environments are not considered. Obviously, the membrane with symmetric (material and thickness) layer structure like

Structure 7. Material sequence: A-B-C-B-A

$$
\text { Layer thickness: } 12321 \quad \text { Total: } 9
$$


will exhibit no dependence on the environmental differences on its opposite sides, and it can be reversed resulting in the same transmission rates of the permeant in question.

It is clear from the examples above that if we vary only one or several (but not all) layer thicknesses to satisfy some new design goals then we change the structural identity of the new film compared to the original (rather than its thickness only). The attempt to use the total permeability $P_{T}$ in Eqs. (7) or (8) even for simple engineering tasks involving structural changes will then lead to incorrect results. Later in the article examples 2 and 3 demonstrate inapplicability of the TR normalization assumption to structurally different films.

In real world each layer has a specific practical purpose such as gas barrier, water vapour barrier, adhesive, food contact layer, etc., and that fact does not warrant a change in its thickness as we vary thickness of one particular layer. In other words the change in one layer thickness to meet a practical goal usually does not involve changing the thickness of other layers. Thus, in engineering practice we are not interested in maintaining the structural identity of the film as we improve its structural design. Therefore the stated TR normalization assumption is invalid in practice since it applies only to structurally identical films. There are also technological and practical limitations to arbitrarily changing layer thickness, e.g., the adhesive layer thickness (usually about 0.3 - 1.0 mil in packaging films) cannot be significantly reduced due to co-extrusion process limitations and loss of interlayer adhesion nor can it be increased due to deterioration of mechanical properties and economic reasons.

\section{General discussion}

To illustrate the point made above let us consider an example calculation in ref. [1] taken from the Handbook of Package Engineering by Hanlon et al. [4].

\section{Example 1}

Tab. 1. Three-layer laminate of the following structure is considered:

\begin{tabular}{ccccc}
\hline Layer & Materials & $L /$ mil & OTR & $P$ \\
\hline 1 & Material 1 & 1.5 & 500 & 750 \\
2 & PVDC & 0.5 & 0.05 & 0.025 \\
3 & Material 2 & 2.0 & 1200 & 2400
\end{tabular}

Here OTR is the oxygen transmission rate in $\mathrm{cc} /\left(100 \mathrm{in}^{2} \cdot 24 \mathrm{~h} \cdot \mathrm{atm}\right)$ at $77^{\circ} \mathrm{F}\left(25^{\circ} \mathrm{C}\right)$ and $50 \% \mathrm{RH}$ and pressure gradient of 1 atm of pure oxygen across the membrane, and $P$ is the material oxygen permeability in $\mathrm{cc} \cdot \mathrm{mil} /\left(100 \mathrm{in}^{2} \cdot 24 \mathrm{~h} \cdot \mathrm{atm}\right)$ at the same conditions added by us for reference using Eq. (2). The permeated oxygen amount is expressed in $\mathrm{cc}\left(\mathrm{cm}^{3}\right)$ at STP.

What are $T R_{T}$ and $P_{T}$ of this laminate? We would want to know both its actual field performance and permeability value on 1 mil basis for comparison with published data for other structures. Using Eq. (3) we correctly determine the total oxygen transmission rate through this laminate (4 mil thick) as 
$T R_{T}=0.05 \mathrm{cc} /\left(100 \mathrm{in}^{2} \cdot 24 \mathrm{~h} \cdot \mathrm{atm}\right)$

If we try to calculate the permeability of this laminate using Eq. (8), we obtain

$P_{T}=0.20 \mathrm{cc} \cdot \mathrm{mil} /\left(100 \mathrm{in}^{2} \cdot 24 \mathrm{~h} \cdot \mathrm{atm}\right)$

which is exactly the same result as reported in ref. [1]. What is the value of this result? Presumably we can compare our laminate with another material or multi-layer structure on 1 mil to 1 mil basis and conclude that one outperforms another, however this comparison is meaningless. The reason is simple: single polymer material can be processed into film of almost any thickness, but our laminate is a prefabricated article with thickness of 4 mil. The same laminate 1 mil thick with proportional reduction of each layer thickness to maintain its structural identity and hence its predicted $P_{T}=$ $0.20 \mathrm{cc} \cdot \mathrm{mil} / 100 \mathrm{in}^{2} \cdot 24 \mathrm{~h} \cdot$ atm simply is not available and quite possibly cannot be made at a reasonable cost if at all (it has to have the following structure: $0.375 \mathrm{mil}$ Material $1+0.125$ mil PVDC +0.5 mil Material 2). Thus, any speculation about its normalized properties is in vain until such a hypothetical structure materializes.

A careful practitioner will avoid such a pitfall simply by using Eqs. (3) and (6) for TR calculation and comparison of multi-layer structures instead of trying to compare meaningless normalized properties like $P_{T}$ according to Eqs. (7) or (8). But it is one thing to report such data for comparison purposes (no matter how confusing) and quite another to base package design decisions on such calculations. The real danger lies in the attempt to use Eqs. (7) and (8) for solving practical engineering problems as demonstrated in the following examples.

\section{Example 2}

Consider the same structure as in Tab. 1, but now our task is to reduce laminate OTR by $50 \%$ by increasing PVDC layer thickness. What will be the new PVDC layer thickness to achieve that goal? In this simple scenario it is intuitively obvious that since PVDC provides nearly $100 \%$ of laminate barrier properties, we need to increase its thickness two fold to achieve the desired total transmission rate reduction. This fact is easily demonstrated using Eq. (3):

$$
T R^{\prime}=\frac{1}{\frac{1}{T R_{1}}+\frac{1}{T R_{2}^{\prime}}+\frac{1}{T R_{3}}}
$$

where $T R^{\prime}{ }_{2}$ and corresponding PVDC layer thickness $L_{2}$ are unknown, while the new target transmission rate is obtained from result (9):

$T R^{\prime}{ }_{T}=0.5 T R_{T}=0.025 \mathrm{cc} /\left(100 \mathrm{in}^{2} \cdot 24 \mathrm{~h} \cdot \mathrm{atm}\right)$

Using Eq. (12) and solving Eq. (11) for $T R_{2}^{\prime}$ we obtain

$T R^{\prime}{ }^{\prime}=\frac{1}{\frac{1}{0.5 \cdot T R_{T}}-\left(\frac{1}{T R_{1}}+\frac{1}{T R_{3}}\right)}$

or $T R_{2}^{\prime} \approx 0.5 T R_{T}=T R_{T}^{\prime}=0.025 \mathrm{cc} /\left(100 \mathrm{in}^{2} \cdot 24 \mathrm{~h} \cdot \mathrm{atm}\right)$.

Then, to obtain $L_{2}$ we use Eq. (2):

$L_{2}^{\prime}=P_{2} / T R_{2}^{\prime}$

resulting in $L_{2}^{\prime}=1.0$ mil. The new laminate structure is 4.5 mil thick: 


\begin{tabular}{lll}
\hline Layer & Material & $L /$ mil \\
\hline 1 & Material 1 & 1.5 \\
2 & PVDC & 1.0 \\
3 & Material 2 & 2.0
\end{tabular}

which is exactly the result we expected. That confirms the validity of Eq. (3) for package design purposes.

Let us now try Eq. (7) to reproduce this result. We denote the increase in layer 2 thickness as $\Delta L$, then the unknown new PVDC layer thickness is

$L_{2}^{\prime}=L_{2}+\Delta L$

Now we attempt to reduce the total permeability $P_{T}$ of the laminate by $50 \%$ in a manner similar to Example 1 (presumably to improve the total transmission rate of the laminate by $50 \%$ as an unstated goal) taking into account the increase in both $L_{2}$ and $L_{T}$ by $\Delta L$ :

$$
P_{T}^{\prime}=0.5 \cdot P_{T}=\frac{L_{T}+\Delta L}{\frac{L_{1}}{P_{1}}+\frac{L_{2}+\Delta L}{P_{2}}+\frac{L_{3}}{P_{3}}}
$$

Solving Eq. (16) for $\Delta L$ we obtain

$$
\Delta L=\frac{L_{T}}{P_{T} \cdot\left(\frac{1}{P_{2}}-\frac{1}{0.5 \cdot P_{T}}\right)}
$$

or $L=L_{T} / 6=0.667$ mil and, correspondingly, new $L_{2}^{\prime}=1.167$ mil. This result is quite different from the earlier obtained 1.0 mil, however when we substitute formula (17) or its numerical solution into Eq. (16) we see that it is the correct solution of the problem as stated. When we calculate the total transmission rate of this new laminate using Eq. (3), the result is

$$
T R^{\prime}=0.0214 \mathrm{cc} /\left(100 \mathrm{in}^{2} \cdot 24 \mathrm{~h} \cdot \mathrm{atm}\right)
$$

which is a $57 \%$ reduction in laminate total OTR and is $14 \%$ below the target OTR. Let us try another structure from practical applications to check whether this discrepancy is always small and can be neglected in engineering applications.

\section{Example 3}

Tab. 2. The following laminate is considered:

\begin{tabular}{ccccc}
\hline Layer & Materials & $L /$ mil & OTR & $P$ \\
\hline 1 & Material 1 & 1.5 & 80 & 120 \\
2 & Material 2 & 1.5 & 0.016 & 0.024 \\
3 & Material 3 & 1.0 & 1000 & 1000
\end{tabular}


at some temperature and relative humidity. The engineering goal is the same: to reduce structural OTR by $50 \%$ by changing the thickness of (primary oxygen barrier) layer 2. From Eq. (3), the total transmission rate of this 4 mil thick structure is

$T R_{T}=0.016 \mathrm{cc} /\left(100 \mathrm{in}^{2} \cdot 24 \mathrm{~h} \cdot \mathrm{atm}\right)$

while its permeability is found using Eq. (8):

$P_{T}=0.064 \mathrm{cc} \cdot \mathrm{mil} /\left(100 \mathrm{in}^{2} \cdot 24 \mathrm{~h} \cdot \mathrm{atm}\right)$

Using result (13), the new transmission rate of layer 2 is

$T R_{2}^{\prime} \approx 0.5 T R_{T}=T R_{T}^{\prime}=0.008 \mathrm{cc} /\left(100 \mathrm{in}^{2} \cdot 24 \mathrm{~h} \cdot \mathrm{atm}\right)$

and the corresponding new layer 2 thickness is

$L_{2}^{\prime}=P_{2} / T R_{2}^{\prime}=3.0 \mathrm{mil}$

This is the expected correct result that suggests to double the thickness of the primary barrier layer. Now we try to use Eq. (7) to reproduce it. Using Eqs. (15) and (16) we attempt to reduce the total permeability $P_{T}$ of the structure by $50 \%$ to obtain

$P^{\prime}{ }_{T}=0.5 P_{T}=0.032 \mathrm{cc} \cdot \mathrm{mil} /\left(100 \mathrm{in}^{2} \cdot 24 \mathrm{~h} \cdot \mathrm{atm}\right)$

leading to result (17) for the layer 2 thickness increase $\Delta L=6 \mathrm{mil}$, and, correspondingly,

$L_{2}^{\prime}=L_{2}+\Delta L=7.5 \mathrm{mil}$

When we calculate $T R^{\prime}{ }_{T}$ of the new structure using Eq. (3), the result is

$T^{\prime}{ }_{T}=0.0032 \mathrm{cc} /\left(100 \mathrm{in}^{2} \cdot 24 \mathrm{~h} \cdot \mathrm{atm}\right)$

which is an $80 \%$ reduction in the total structure OTR and $60 \%$ below target OTR enormous overkill compared to the stated goal also implying prohibitive material waste.

Let us understand what went wrong when we used Eq. (7). In attempt to reduce the permeability $P_{T}$ of the total structure, i.e., normalized $T R_{T}$, we designed a laminate that on 1 mil thickness basis is $50 \%$ better than the old one with the implicit assumption of proportional reduction of each layer thickness to maintain its structural identity. In example 2, when the actual redesigned 4.667 mil thick laminate was analyzed we saw that its real world performance $\left(T R_{T}\right)$ was improved by $57 \%$ due to thicker than necessary PVDC layer. Thus, we had to waste unnecessary material because of the incorrectly stated goal of improving $P_{T}$ of reference 1 mil structure suggested by Eq. (7) instead of $T R_{T}$. The discrepancy in example 3 was even more pronounced. The point made earlier, however, is that no such ideal 1 mil thick 'reference' structure exists nor it is practical to make for any purpose. The actual laminate will never be 1 mil thick since it is impossible to reduce all layer thicknesses without compromising the useful properties of this film such as mechanical properties, regulatory limitations on food contact layer thickness, etc. That supports our earlier assertion that it is meaningless to compare multi-layer structures on basis of their 'permeability' or transmission rates normalized to unit thickness.

The correct way to state the original design problem and utilize the concept of 'permeability' of heterogeneous layered structure should have been: how to reduce the overall OTR of the laminate by $50 \%$ by proportionally changing the thickness of all layers, rather than the primary barrier layer. Now we deal with structurally identical films and the answer is trivial: double the total film thickness by doubling the thickness of all layers. However, practical experience leads us to the conclusion that 
the layer thickness selection is independent from each other since each layer in a package structure serves a different purpose. The situation in which all layer thicknesses are proportionally changed to maintain the structural identity of the film does not correspond to real world development efforts since the goal of structural design improvement can be formulated as changing the structural identity of the existing design to achieve a new level of performance. Thus, maybe it is time to stop reporting 'permeability' values for multi-layer and other heterogeneous structures that are not structurally identical as this approach is clearly misleading.

The same problem arises when we attempt to use Eq. (8) for solving examples 2 and 3 since it is not $P_{T}$ that must be routinely improved but $T R_{T}$. Therefore, any equation where $P_{T}$ of multi-layer structure is used for calculation of film component properties will produce incorrect results unless the structural identity of the modified structure is strictly maintained, and that is simply not practical and leads to confusion. In fact, new ASTM Standards D1434-82 (updated in 1998) and D3985-02, respectively, for gas and oxygen permeability testing of plastic film and sheeting specifically refer to permeability only when homogeneous materials are considered [2]. For heterogeneous structures only gas transmission rates can be determined. It is also worth noting that the authoritative references and textbooks on packaging engineering and technology [4-7] and even monographs on diffusion mathematics [8] routinely use Eqs. (7) and (8) in discussing permeability of layered heterogeneous structures further clouding the presented concepts.

Another implication of using Eqs. (7) and (8) for engineering purposes is that the resulting Eq. (16) may lead to physically meaningless results (division by zero) at some combinations of parameters. That situation is never present when Eqs. (3) or (6) are used for TR reduction purpose. Of course, if one attempts to increase $T R_{T}$ by varying one layer thickness, meaningless results are possible with Eqs. (3) and (6) since other layers may provide sufficient barrier to permeation to prevent $T R_{T}$ increase to a target value even with zero thickness of the selected layer. In the following section we will discuss general limitations of the steady state transmission rate approach and will refer only to Eqs. (3) and (6) as relevant for package design purposes.

\section{Other limitations}

Eqs. (3) and (6) are derived from Fick's first law of diffusion relating flux of the matter $J_{x}$ to its concentration gradient (more precisely, the gradient of chemical potential of the diffusing species) in $x$ direction:

$$
J_{x}=-D \frac{d c}{d x}
$$

which can be considered a steady state or instantaneous case of Fick's second law of diffusion relating concentration change in time to spatial concentration gradients (one dimensional version with concentration independent diffusivity $D$ is shown):

$$
\frac{\partial c}{\partial t}=D \frac{\partial^{2} c}{\partial x^{2}}
$$

Here $c=c(x, t)=[\mathrm{A}]_{M}$ is the concentration of the permeating species $\mathrm{A}$ in the material $\mathrm{M}$, and $D \equiv D_{A M}$ is the diffusivity of permeant $\mathrm{A}$ in material $\mathrm{M}$ assumed to be independent of concentration $c$. The permeability $P$ of a gas in a solid is defined as a product of the gas diffusivity $D$ and its solubility coefficient $S$ in the solid matrix: 
That follows from the solution-diffusion mechanism of permeation originally proposed by Graham [9]. The book by Vieth [10] provides a more comprehensive review of gas and vapour diffusion mechanisms in different types of polymers although it stops short of deriving equations analogous to (3) and (6) for multi-layer structures. Eqs. (26) and (27) deal with the permeant concentrations in the material; hence to convert the concentrations to partial gas pressures outside the membrane Henry's law may be used:

$$
c=S \cdot p
$$

The solubility coefficient $S=S_{p, T}$ of gaseous permeant $A$ in material $M$ is the equilibrium thermodynamic property relating the equilibrium permeant concentration in the material to its partial pressure in the adjacent gas phase:

$$
S_{p, T} \equiv\left(S_{M}^{A}\right)_{p, T}=\frac{[A]_{M}}{p_{A, g a s}}
$$

The permeant is said to obey Henry's law if $S_{p, T}$ as defined in Eq. (30) is constant for the range of pressures of interest from 0 to $p^{*}$.

The correct way to treat solubility of gases in solid media in direct contact with each other as present in multi-layer polymer structures is to introduce the partition coefficient $H$ of the permeant between these media as a ratio of equilibrium concentrations of the permeant $\mathrm{A}$ in materials $\mathrm{M}$ and $\mathrm{N}$ :

$$
H \equiv H_{M N}^{A}=\frac{[A]_{M}}{[A]_{N}}
$$

which in general depends on absolute values of permeant pressures in the materials. Due to low solubility of gases in most polymers, the gas-polymer solutions can be considered dilute and Henry's law applies in most cases. Since partial pressures (more accurately, activities) of A have to be the same in both materials if they are in equilibrium, the concentrations in Eq. (31) can be replaced by respective solubilities of $A$ in materials $M$ and $N$ using Henry's law (29):

$$
H_{M N}^{A}=\frac{S_{M}}{S_{N}}
$$

There are few notes to be made regarding the applicability of these equations in the general sense. Material and gas interaction properties $D$ and $S$ depend on temperature, and for most materials the regular Van't Hoft-Arrhenius dependence can be assumed [11]:

$$
\begin{aligned}
& D=D_{0} \exp \left(\frac{-E_{D}}{R T}\right) \\
& S=S_{0} \exp \left(\frac{-\Delta H_{S}}{R T}\right)
\end{aligned}
$$

where $E_{D}$ is the activation energy of diffusion, and $\Delta H_{S}$ is the molar heat of sorption. If the concentration of permeant in a gas phase in direct contact with the solid is $c_{0}$, then the steady state concentration of the permeant $c_{1}$ in the solid right across the phase boundary is 
$c_{1}=c_{0} \exp \left(\frac{-\Delta H_{S}}{R T}\right)$

This type of dependence is valid unless the solid material undergoes physical changes when the temperature $T$ is altered. Glass transition, change in crystallinity, melting, deformation, and relaxation of molecular orientation imposed by a processing technique are several examples of such changes in polymer systems. Even at the same temperature, different crystallinity and morphological structure of a polymer imparted by the processing method are some of the reasons why the measured permeability of an annealed injection molded plaque might be different from that of a rapidly cooled film of the same thickness extruded with a high draw ratio. While viscoelasticity of solid polymers manifesting itself in time-dependent responses to deformation and relaxation of molecular structure far from equilibrium is a widely studied area [12-14], its effects on transport properties of polymers especially related to oxygen and water vapour diffusion are largely unknown [10]. In case of very thin films, the sorption properties of the film surface and structural defects (like pinholes) may also introduce nonlinearity of the transmission rate dependence on the film thickness.

Apart from Van't Hoft-Arrhenius dependence of $D$ and $S$ on temperature, the diffusivity $D$ of a particular gas is known to be affected by the presence of co-permeants (if a mixture of different gases is present), while the solubility $S$ is often a nonlinear function of external pressure, and there is a solubility limit for many polymer-gas systems at high pressures [10,11]. The linear relationship suggested by Henry's law and the diffusivity independence of the sorbed permeant concentration in Fick's first law hold only when 'permanent' gases like nitrogen, oxygen, hydrogen and carbon dioxide are permeants, and the gas pressure is nearly atmospheric. When interactions of gas with polymer become important, other types of sorption are often observed like Langmuir, Flory-Huggins and BET sorption isotherms [11]. The sorption isotherm type greatly affects the permeation behaviour as a function of external pressure. Therefore caution should always be exercised when calculating total transmission rates using Eqs. (3) and (6) at conditions far from those at which transmission rates for individual structural layers were obtained, or different processing methods were used in manufacturing multi-layer structures and homogeneous material samples for transmission rate measurements.

A note is due on homogeneity of materials for transmission rate measurement. With rapid development and commercialization of heterogeneous materials like polymer composites filled with inorganic particulates, we need to distinguish between heterogeneous materials and heterogeneous structures. All preceding discussion dealt with layered structures, i.e., the heterogeneous structures consisting of clearly defined distinct layers made from different materials. The structure of the material forming a single layer was not considered, and its permeability and transmission rate for the specific permeating species were treated as given. While it is true that polymer composites may exhibit gas and vapour transmission rates much different from those of an unfilled polymer matrix, this approach to permeability of a single layer is still valid if the particulate is finely (and, in a sense, 'homogeneously') dispersed in the polymer matrix. More precisely, if

a) the characteristic size of the dispersed particles is much smaller than the layer thickness

b) the particles are dispersed randomly without discernible agglomeration in the matrix 
c) the volume fractions of the dispersed particles are the same in bulk polymer and at the layer surfaces

d) particle orientation in bulk and at the layer surfaces is the same, e.g., random or oriented (orientation is essential only if the particles possess quantifiable uniformly irregular shapes, e.g., constant high aspect ratio circular or rectangular platelets)

then we may consider the composite material as 'homogeneous' for permeability purposes and use its gas transport properties in multi-layer film equations (with the corresponding sorption isotherm behaviour changes due to filler presence, if any). 'Homogeneous' for permeability purposes simply means that with all other properties being constant, any change in the layer thickness without violating conditions a - $d$ will result in the inversely proportional change in the measured transmission rates according to Eq. (1) and satisfying Eq. (2). In case of high aspect ratio particles in clay filled nanocomposites, particle orientation relative to layer thickness direction will significantly affect measured transmission rates. Such a material even if still considered 'homogeneous' will not be isotropic for gas permeation. Hence, all gas transport properties exhibited by it will be diffusion direction specific: that should be taken into account when using single layer permeability data for analysis of gas transmission through a multi-layer structure containing such composite materials.

Acknowledgement: Authors thank Prof. Witold Brostow of the University of North Texas and the manuscripts reviewers for useful comments and suggestions on the scope of presentation.

[1] Cooksey, L.; Marsh, K. S.; Doar, L. H.; Food Technol. 1999, 53, 60.

[2] ASTM, Annual Book of Standards 15.09. Paper; Packaging; Flexible Barrier Materials; Business Imaging Products, ASTM, Philadelphia, PA 2003.

[3] Massey, L. K.; "Permeability Properties of Plastics and Elastomers", $2^{\text {nd }}$ edition, Plastics Design Library, Norwich, NY 2003.

[4] Hanlon, J. F.; Kelsey, R. J.; Forcino, H. E.; "Handbook of Package Engineering", $3^{\text {rd }}$ edition, Technomic Publishing Co., Lancaster, PA 1998, p. 106.

[5] Salame, M.; 'Barrier Polymers', in: Wiley Encyclopedia of Packaging Technology, Baker, M., editor; Wiley, New York 1986, p. 52.

[6] Delassus, P.; 'Barrier Polymers', in: Wiley Encyclopedia of Packaging Technology, $2^{\text {nd }}$ edition, Brody, A.; Marsh K. S.; editors; Wiley, New York 1997, p. 76.

[7] Robertson, G. L.; "Food Packaging Principles and Practice”, Marcel Dekker, New York 1993, p. 100.

[8] Crank, J.; "The Mathematics of Diffusion", $2^{\text {nd }}$ edition, Oxford University Press, Oxford 1975, p. 267.

[9] Graham, T.; Philos. Mag. 1866, 32, 401.

[10] Vieth, W. R.; "Diffusion In and Through Polymers", Hanser, München 1991, pp. 78.

[11] van Krevelen, D. W.; "Properties of Polymers", $3^{\text {rd }}$ edition, Elsevier, Amsterdam 1990.

[12] Ward, I. M.; "Mechanical Properties of Solid Polymers”, Wiley, New York 1971. 
[13] Goldman, A. Y.; "Prediction of the Deformation Properties of Polymeric and Composite Materials", ACS, Washington DC 1994.

[14] Goldman, A. Y.; 'Viscoelasticity, Creep and Stress Relaxation', in "Performance of Plastics", Brostow, W., editor; Hanser, München 2001, p. 121. 